Sensors 2003, 3, 361-370

sensors

ISSN 1424-8220

(C) 2003 by MDPI

http://www.mdpi.net/sensors

\title{
Detecting Pesticide Residue by Using Modulating Temperature Over a Single $\mathrm{SnO}_{2}$-Based Gas Sensor
}

\author{
Xingjiu Huang ${ }^{1,2}$, Jinhuai Liu ${ }^{1,3 *}$ Zongxin $\mathrm{Pi}^{1}$ and Zengliang $\mathrm{Yu}^{3}$ \\ ${ }^{1}$ Hefei Institute of Intelligent Machines, Chinese Academy of Sciences, Hefei, 230031, P. R. China \\ ${ }^{2}$ Department of Chemistry, University of Science and Technology of China, Hefei, 230026, P.R.China \\ ${ }^{3}$ Institute of Plasma Physics, Chinese Academy of Sciences, Hefei, 230031, P.R. China \\ * Author to whom correspondence should be addressed: jhliu@iim.ac.cn
}

Received: 30 May 2003 / Accepted: 23 July 2003 / Published: 11 September 2003

\begin{abstract}
A new rapid detecting method (called dynamic measurements ) was reported to detect and distinguish the presence of two pesticide gases in the ambient atmosphere. The method employed only a single $\mathrm{SnO}_{2}$-based gas sensor in a rectangular temperature mode to perform the qualitative analysis of a binary gas mixture (acephate and trichlorphon) in air. Polar plots was used for quantitative analysis which the feature extraction was performed by FFT. Experimental results showed that high selectivity of the sensor achieved in the range of $250 \sim 300^{\circ} \mathrm{C}$ and modulating frequency $20 \mathrm{mHz}$, one can easily observe the qualitative difference among the response to pure acephate and trichlorphon gases of the same concentration and to the mixture, and the concentration of pesticide gases can be obtained based on the changes of polar plots.
\end{abstract}

Keywords: $\mathrm{SnO}_{2}$ gas sensor; modulating temperature; Pesticide residue

\section{Introduction}

SnO2-based gas sensors had been extensively applied to gases detection [1]. Present well known advantages included their low costs and high sensitivities; well known disadvantages concerned their lack of stability and selectivity[2,3]. In practical applications, several attempts were usually made to 
overcome their disadvantages, by, for example, using chromatographic columns to separate the components, by operating at different temperatures, by choosing different burning-in procedures, dopants, surface chemical modification, measuring frequencies,etc.[1-6]. For common applications of pattern recognition and multi-component analysis of gas mixtures, arrays of sensors $[7,8]$ were usually chosen which operate at constant temperature. In these cases a lack of selectivity and therefore overlapping sensitivities of different sensors was of advantage[9,10].Some authors[11-14] had indicated that temperature modulation of metal-oxide sensors provides more information from a single sensor than isothermal operation, allowing improved research works in gas detection such as $\mathrm{CH} 4$, $\mathrm{CO}, \mathrm{NO} 2$. Several investigations had been focused on dynamic sensor measurements obtaining adequate results, and nevertheless they used non-commercial sensors and often required sophisticated heater control waveforms[15,16].

Pesticide residue had always been the most important problem on food security[17,18]. In recent years, therefore, considerable effort had been directed towards the detecting techniques of pesticide residue[17-22]. Although there had precision of analysis, it was well-known that the disadvantages of the conventional detecting method, such as chromatographic detection, was too slow to detect gas sample in situ and affected the timeliness of the analysis. So more attention had been focused on the research of rapid test method of pesticide residue. At present, the rapid analyzing techniques included immunoassay[19], biosensor[20,21], chromatographic and mass spectrometry[22], polarographic method[23] and soon. A simplified indirect method was developed using adapted versions of molecular emission cavity analysis based on measurements of the intensity of the emission band of indium monochloride at 359.9nm[24], but in the case of the above mentioned methods there existed some different limitation[17]. Meanwhile, there was very few the report about detecting pesticide residue by using $\mathrm{SnO} 2$ sensor.

In the present study, a modulated temperature operation mode was chosen as a new approach to improve the selectivity of a gas sensor to detect pesticide gases in the controlled way, and to reduce the overall power consumption by employing only a single sensor rather than an array. Meanwhile, polar plots was employed to analysis the response characteristics.

\section{Experimental}

The thick film sensors were made by depositing thick films of tin oxide on ceramics substrates[25]. The devices were used according to the reference of [26]. The preparation of the sensitive material was described elsewhere[27]. The grain sizes of the materials were around 20 and $50 \mathrm{~nm}$. The temperature of the sensors was varied between 50 and $300{ }^{\circ} \mathrm{C}$ by a rectangular modulation of the heating voltage with a frequency of $20 \mathrm{mHz}, 25 \mathrm{mHz}, 30 \mathrm{mHz}, 50 \mathrm{mHz}$. The experimental test setup consisted of computer-driven mass flow controllers, a Teflon chamber and a multimeter for measurements in the millisecond range. The temperature was varied by modulating the heating voltage with a power supply (HP 6035A) driven by a frequency generator (HP 3325B). A flow of compressed air was used as

purging gas. Test gases were in the concentration of $0.1 \mathrm{ppm}$ acephate and trichlorphon gas (Analytical standard, provided by Sigma-Aldrich Laborchemikalien Gmbh).

The gas sensitivity was defined : $\mathrm{S}=$ Rair/Rgas, where Rair and Rgas were the resistance of the sensor in air and test pesticide gas respectively. 


\section{Results and discussion}

The static response of the sensor on trichlorphon and acephate gas atmosphere

The responses to $0.1 \mathrm{ppm}$ trichlorphon, acephate gas and $0.2 \mathrm{ppm}$ mixture at $300^{\circ} \mathrm{C}$ for $\mathrm{SnO}_{2}$ sensor were reported in Fig.1. the response time defines the time taken for the sensor to reach $90 \%$ of the saturation value after contacting by the test gas with the surface of the sensor. In Fig.1, one can clearly observe that the sensor exhibit the rapid response upon exposure to the trichlorphon and acephate gas, we noticed that the response time to both acephate and trichlorphon gas or to $0.2 \mathrm{ppm}$ mixture was only several seconds. It also showed that, however, in addition to the changes of resistance and response time there was no other information about reaction process. In particular, there was no reaction

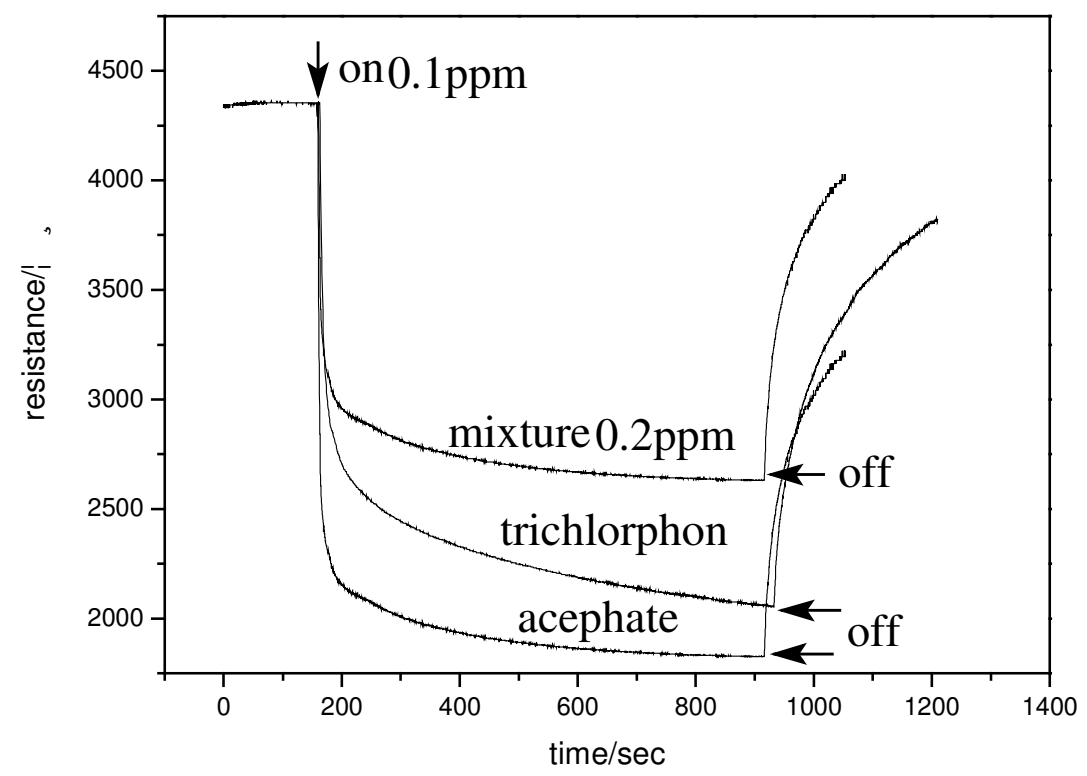

Figure 1. Static response characteristics of the sensor to trichlorphon, acephate and mixture gas atmosphere (operating temperature: $300{ }^{\circ} \mathrm{C}$ )

phenomenon but declining of element resistance by comparing a single pesticide gas, this case can be explained by the fact that the concentration of mixture was larger than that of a single gas. That is to say, during static measurements, we only observed the resistance changes of sensing element of initial and final state, in the case of the other changes during reaction process we did not obtain any other information.

\section{Effect of temperature on the sensitivity}

Fig.2 was a plot of the sensitivity of the sensor as a function of a constant concentration of acephate, trichlorphon and mixture gas at different operation temperatures. It was evident that the sensor exhibited a significant increase in sensitivity upon exposure to a very low concentration of 
acephate and trichlorphon and mixture gas, at round $300{ }^{\circ} \mathrm{C}$ the sensitivity reached a maximum, and decreased with the increasing of operation temperature subsequently.

\section{Effect of temperature modulating frequency on the response of test gas}

Figs. 3 and 4 clearly showed the time-dependence changes shape of resistance of the sensor in the presence of trichlorphon and acephate gas in air under different temperature modulating frequency by controlling temperature range of $250 \sim 300{ }^{\circ} \mathrm{C}$. Obviously, temperature modulating frequency had a significant effect on the sensing behaviour of the sensor. With the decrease of modulating frequency we can clearly observe that the sensing characteristics of the sensor had been exhibited to whether

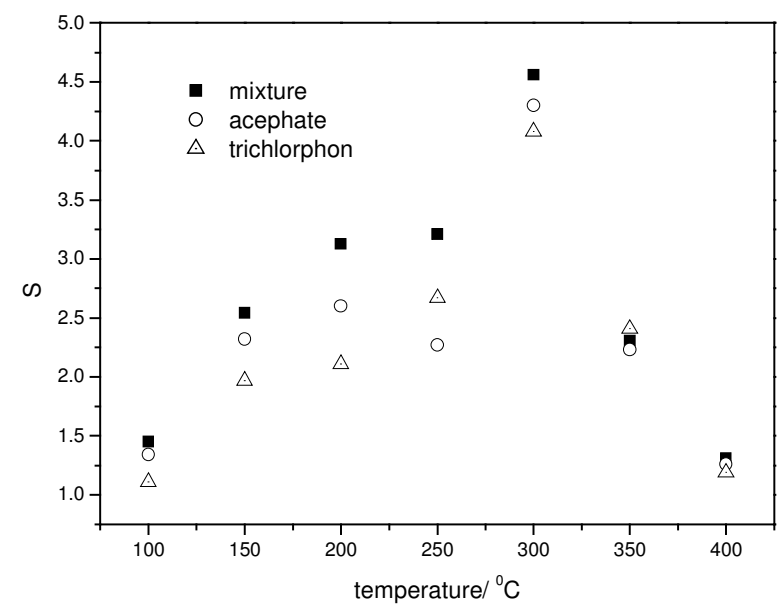

Figure 2. The sensitivity as a function of $0.1 \mathrm{ppm}$ trichlorphon, acephate gas and $0.2 \mathrm{ppm}$ mixture respectively at different temperature

acephate or trichlorphon gas. Meanwhile, other experimental results (frequency $<20 \mathrm{mHz}$ ) showed that there were no changes of sensing characteristics.

Effect of temperature under constant frequency $20 \mathrm{mHz}$

To optimize the selectivity of a temperature modulated sensor, it was necessary to obtain a relationship between a given temperature and its conductance response in the presence of specific gas. Fig.5 reported the effect of temperature on the response of pesticide residue gas, for example, $0.1 \mathrm{ppm}$ trichlorphon under a constant modulating frequency $20 \mathrm{mHz}$. From the figure, one can easily observe the evident different signal by different temperature ranges. This case noted that the sensor exhibited enhanced selectivity to trichlorphon gas with temperature increasing. Combining with optimum temperature in this study, we suggested that the trichlorphon gas was identified by means of a relative complete response signal in the temperature modulating range of $250 \sim 300{ }^{\circ} \mathrm{C}$. As the sensor cooled, we found that the resistance not only increased but decreased, Also, upon heating, the resistance not only decreased but increased. Obviously, there were different surface reaction mechanism between the trichlorphon and chemisorbed oxygen under different temperature ranges. 

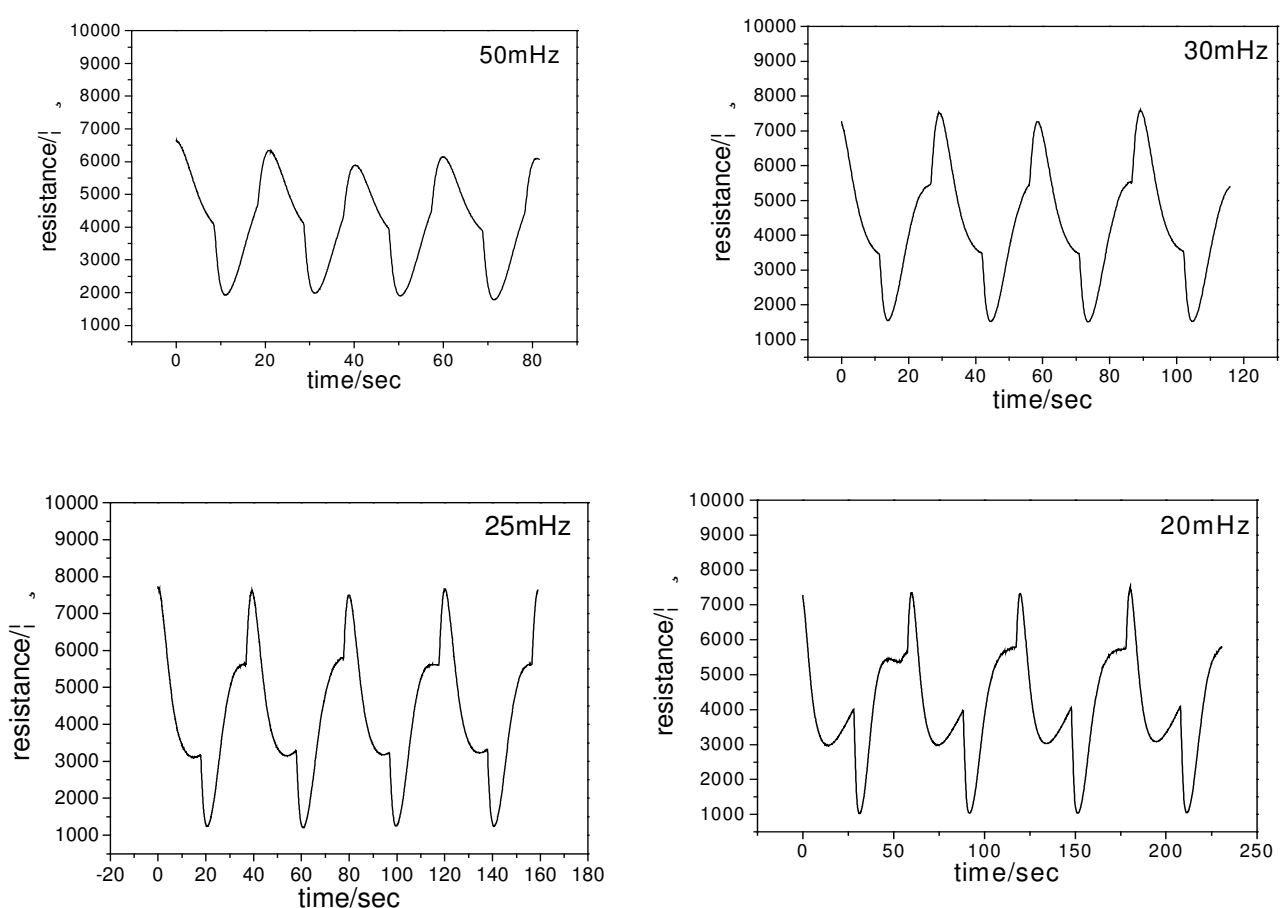

Figure 3. Effect of modulated temperature frequency on the response of $0.1 \mathrm{ppm}$ trichlorphon
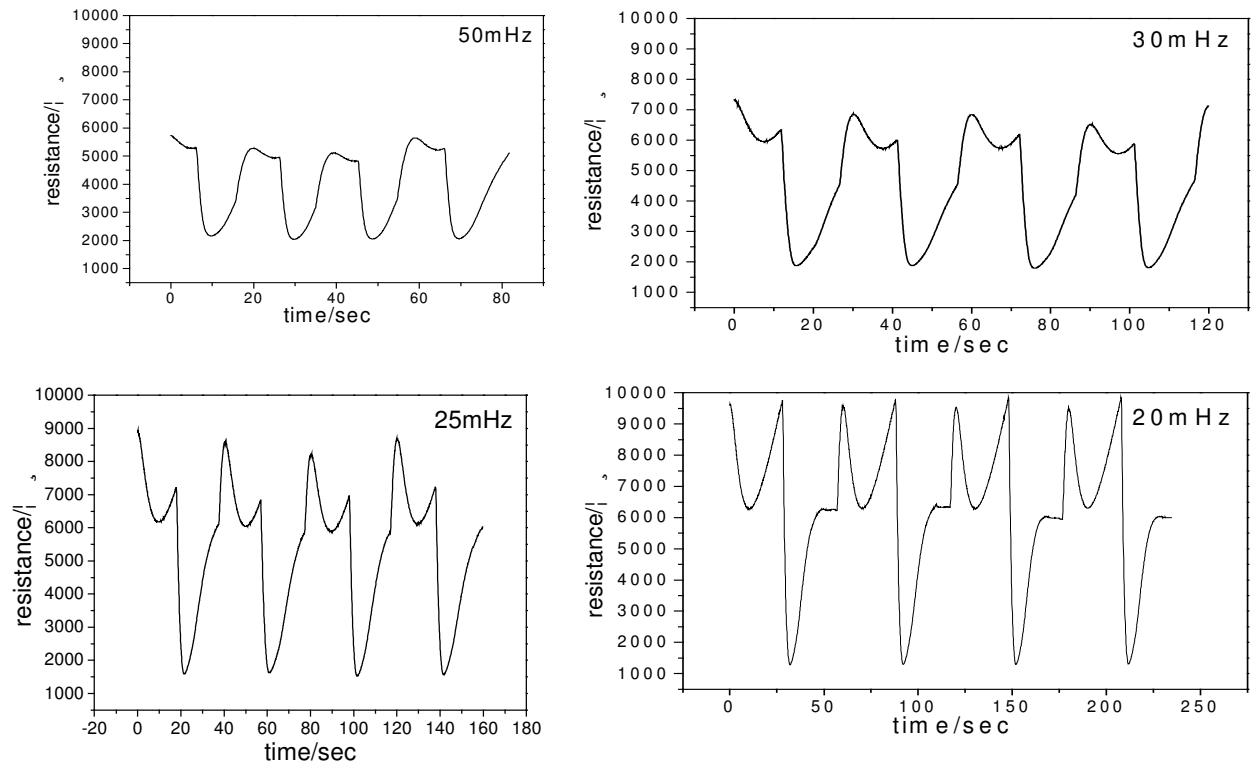

Figure 4. Effect of modulated temperature frequency on the response of $0.1 \mathrm{ppm}$ acephate 

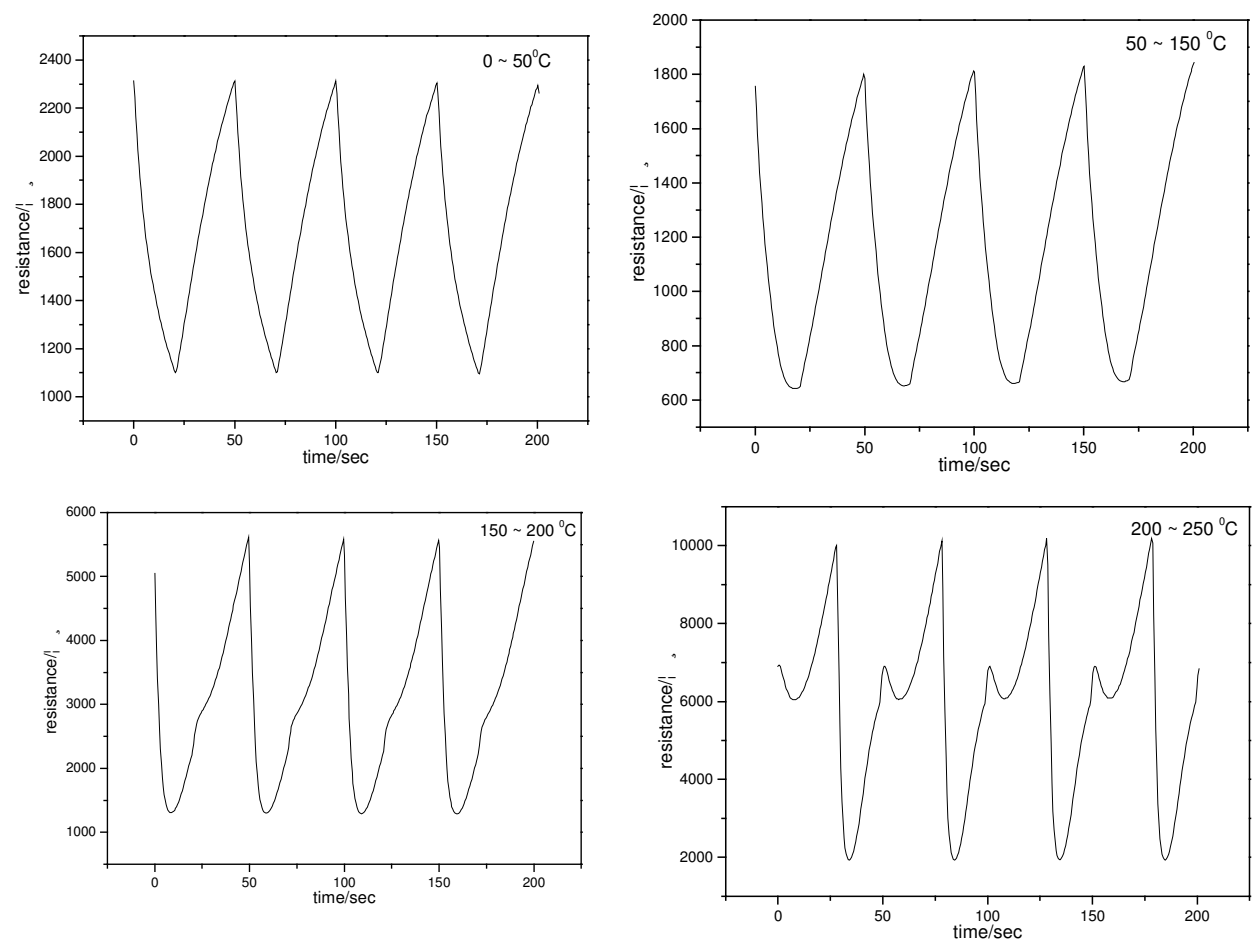

Figure 5. Effect of temperature on the response of $0.1 \mathrm{ppm}$ trichlorphon gas under frequency $20 \mathrm{mHz}$

\section{Data evaluation and feature extraction}

Fig.6 showed the resistance versus temperature curves for air, acephate gas, trichlorphon gas and mixture of acephate and trichlorphon gas. As can be seen, the curves for the different pesticide gases were apparently different from each other. It was noted that characteristic responses were given with a single sensor by modulating temperature in this study. With the repeated application of a high temperature (i.e. $300{ }^{\circ} \mathrm{C}$ in this study), the adsorbed chemical species may be eliminated from the sensor. This results in repeated cleaning of the sensor and gave us the high reproducibility of the shape of the resistance-temperature curve.

In order to analyze the characteristics of the resistance curves in a quantitative manner, polar plots was used herein which the feature extraction was performed by FFT. The analyzed sensor variables were the amplitudes corresponding to the resistance offset (corresponding frequency $=0 \mathrm{~Hz}$ ), the fundamental frequency, which was in our case the frequency of the temperature modulation $(20 \mathrm{mHz})$, and the first four harmonics. Fig.7 showed the way in which the polar plots were constructed, i.e. the represented values being obtained by the normalization of the above listed amplitudes in the test gases to the amplitudes in reference air. In Figs 8 and 9, typical polar plots were given for the two different pesticide gases and mixture. One can clearly distinguish between the cases in which one or the other of the target gases were present in the test atmosphere. In this way on can perform a identification of both gases. 

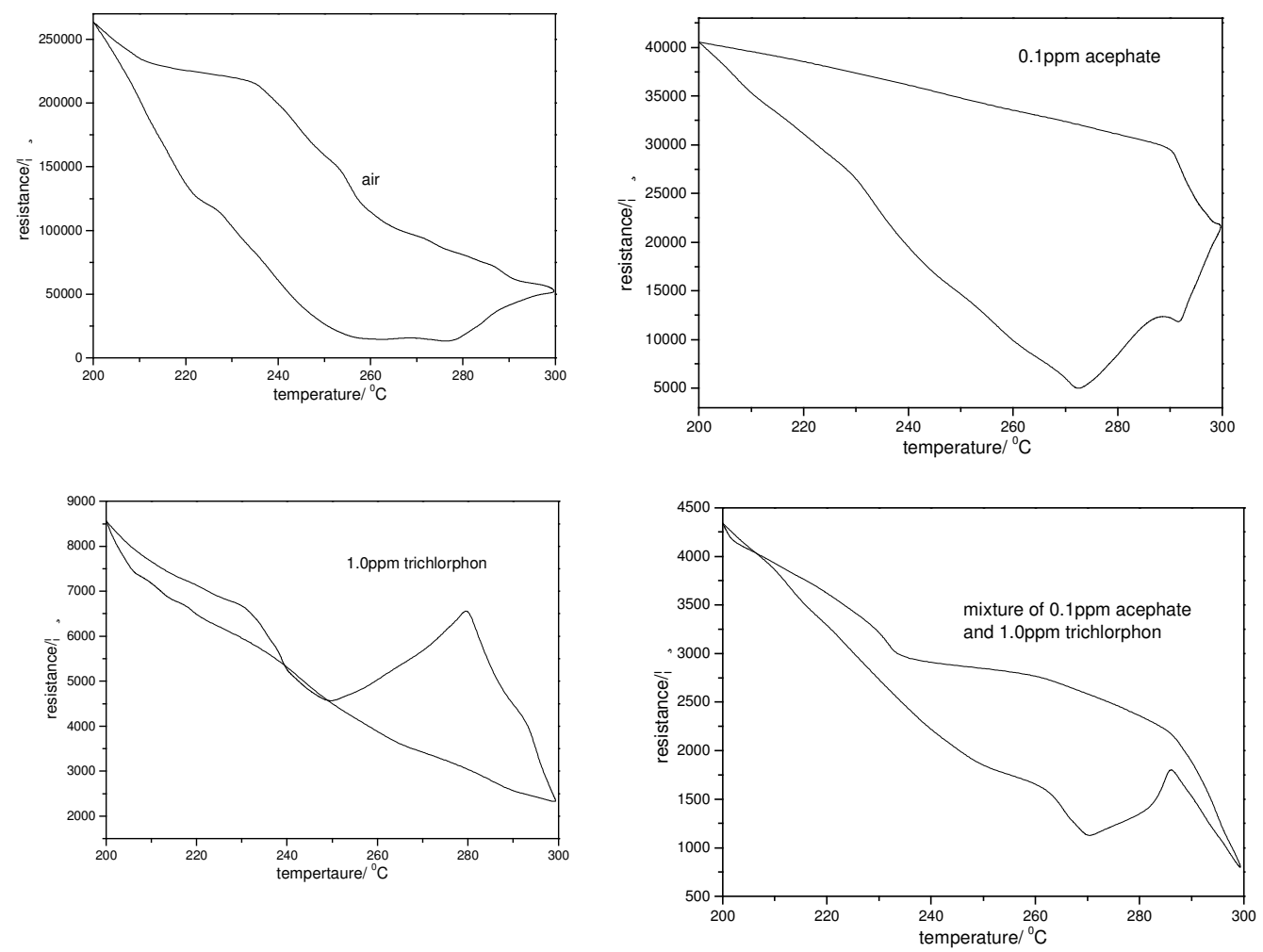

Figure 6. Characteristic response of resistance versus temperature curve for air, $0.1 \mathrm{ppm}$ acephate, $1.0 \mathrm{ppm}$ trichlorphon and mixture of $0.1 \mathrm{ppm}$ acephate and $1.0 \mathrm{ppm}$ trichlorphon. A square voltage $(\mathrm{f}=20 \mathrm{mHz})$ was applied to the heater of the sensor. The temperature was changed approximately between 200 and $300{ }^{\circ} \mathrm{C}$
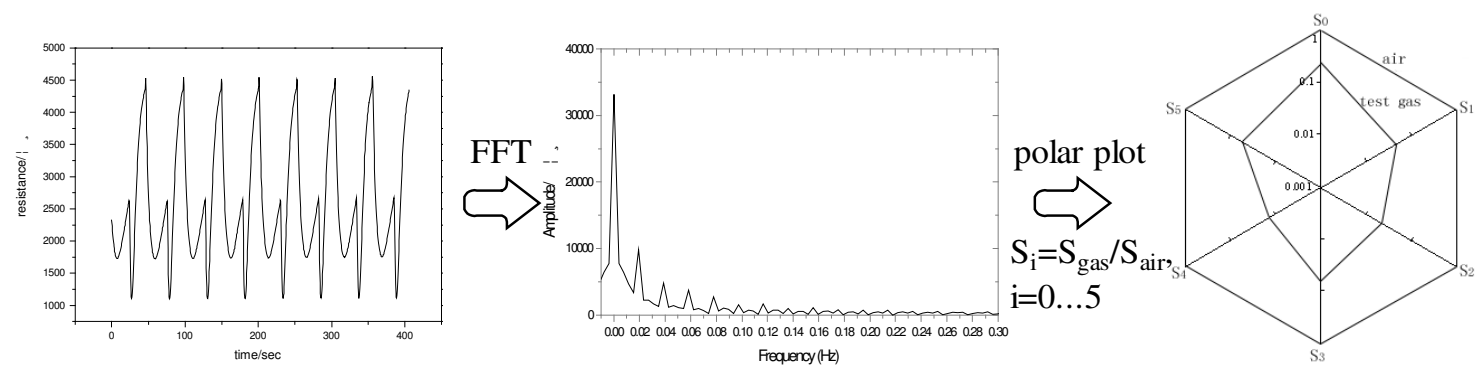

Figure 7. Illustration showing the way in which the polar plots were constructed. Sensor variables used: the amplitude corresponding to $\mathrm{OHz}$ frequency, the amplitude corresponding to the fundamental frequency and the first four harmonics. The values in the polar plot were obtained by normalizing each component to the corresponding amplitude in air.

It was widely accepted that the key process in the response of the semiconductor to a reducing gas involved the modulation of the concentration of adsorbed oxygen species such as $\mathrm{O}^{2-}, \mathrm{O}^{-}$or $\mathrm{O}^{2-}$. By withdrawing electron density from the semiconductor surface, adsorbed oxygen gave rise to Schottky potential barriers at grain boundaries, and thus increased the resistance of the sensor surface. Reducing 
gases decreased the surface oxygen concentration and thus decreased the sensor resistance. The temperature dependence of this process arose in part from the differing stabilities of the surface oxygen species over different temperature ranges.

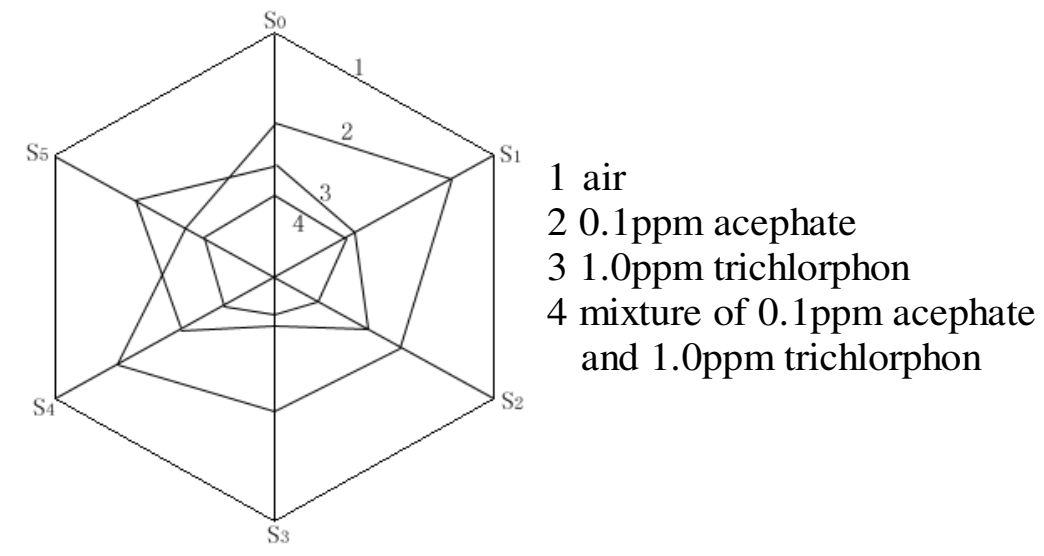

Figure 8. Polar plots of the $0.1 \mathrm{ppm}$ acephate, 1.0ppm trichlorphon and mixture of $0.1 \mathrm{ppm}$ acephate and 1.0ppm trichlorphon gas.
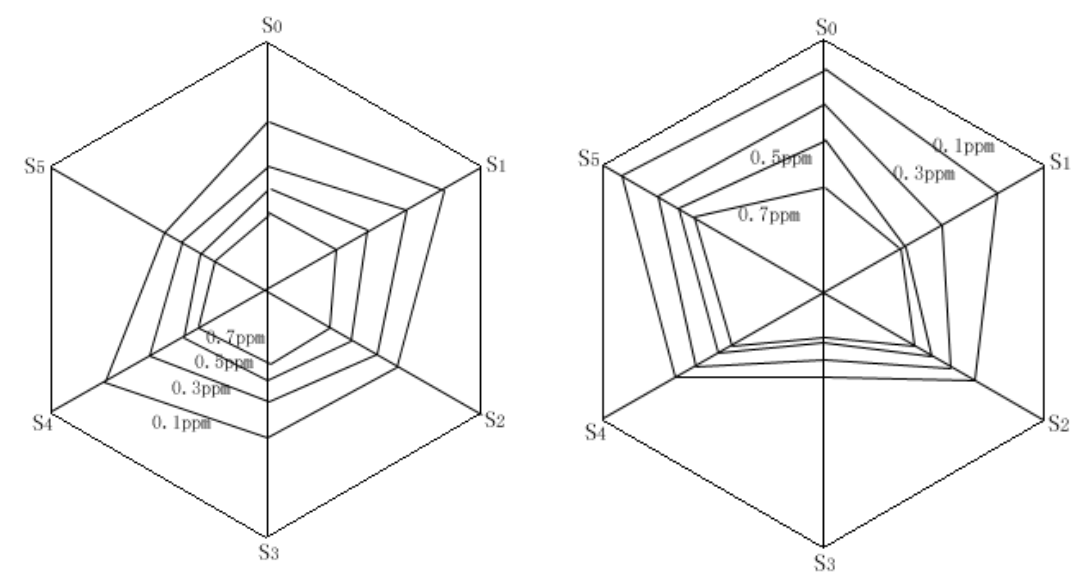

Figure 9. Polar plots of acephate and trichlorphon gas under different concentration (left: acephate, right: trichlorphon)

While the identity of the surface oxygen species remains slightly more controversial, it is clear that different gases have characteristic optimum oxidation temperatures, and therefore give rise to characteristic conductance-temperature profiles.

Sears et al. ${ }^{[28,29]}$ suggested several advantages that can arise from the application of an oscillating heater voltage. (1) a cyclic temperature variation can give a unique signature for each gas due to the different rates of reaction of various test gases at different temperatures. (2) periodic shifted to higher temperatures may be required to clean the sensor surface due to low temperature operation can lead to the accumulation of incompletely oxidized contaminants. (3) thermal cycling can lead to improvements in sensitivity because for each gas there was usually a point (temperature or heater voltage) in the cycle which corresponding to a maximum in the conductance-temperature profile. 


\section{Conclusion}

Experiment found that a very low concentration pesticide gases (acephate and trichlorphon $0.1 \mathrm{ppm}$, respectively) in the ambient atmosphere were rapidly detected and distinguished clearly by using only a single $\mathrm{SnO}_{2}$ sensor operated in the rectangular temperature mode. The optimizing temperature was between 250 and $300{ }^{\circ} \mathrm{C}$ and the optimizing modulating frequency was $20 \mathrm{mHz}$. Experimental data also showed that qualitative and quantitative analysis about pesticide gases can be performed by polar plots. We consider that it will become a significant exploratory development method to rapidly detect pesticide residue gas. Additional studies are now on the way to extend this approach to identify other pesticide residue gas mixtures with more components and to understand the basic physical phenomena. Future work will also be devoted to the development of appropriate feature extraction procedures for this non-linear frequency-time problem.

\section{Acknowledge}

This work was financially supported by the National Natural Science Foundation of China (project 60274061) and Anhui Province Natural Science Foundation (project 01041404), which are gratefully acknowledged.

\section{References}

1. W. Gopel, K.D.Schierbaum, Sensors and Actuators B 1995,26-27,1-12

2. K.Ihokura, J.Watson, Stannic Oxide Gas Sensors, Principles and Applications, CRC press, Boca Raton, FL,1994

3. P.Moseley, B.Tofield, Solid State Gas Sensors, Adam Hilger, Bristol, 1987

4. J.-H.Liu, X.-J.Huang,G.Ye, et al. Sensors, 2003,3,110-118

5. U.Weimar, W.Gopel, Sensors and Actuators B 1995,26,13-18

6. K.Wada, M.Egashira, Sensors and Actuators B 1998,53,147-154

7. T.Maekawa, K.Suzuki, T.Takada, T.Kobayashi, M.Egashira, Sensors and Actuators B 2001,80,5158

8. D.-S.Lee, J.-K Jung, J.-W.Lim, Sensors and Actuators B 2001,77,228-236

9. H.V.Shurmer, J.W.Gardner, Sensors and Actuators B 1992,8,1-11

10. K.D.Schierbaum, U.Weimar, W.Gopel, Sensors and Actuators B 1990,2,71-78

11. A.Ortega, S.Marco, A.Perera, T.Sundic, A.Pardo, J.Samitier, Sensors and Actuator B 2001,78,3239

12. A.Heilig, N.Barasan, U.Weimar, M.Schweizer-Berverich, J.W.Gardner, W.Gopel, Sensors and Actuators B 1997,43, 45-51

13. A.P.Lee, B.J.Reedy, Sensors and Actuators B 1999,60, 35-42

14. JL.Ratton,T.Kunt,et al., Sensors and Actuators B 1997,41,105-120

15. R.E.Cavicchi, J.S.Suehle, K.G.Kreider, M.Gaitan, Sensors and Actuators B 1996,33,143-146

16. M.Schweizer-Berberich,M.Zdralek,U.Weimar,W.Gopel, et al., Sensors and Actuators B 2000,65,91-93

17. C.M.Torres, Y.Pico J.Manes, Journal of Chromatography A, 1996,754,301-331 
18. P.Lea, F.Mladen, Biosensors and Bioelectronics, 2003,18, 1-9

19. V.Z.Anatoliy, B.D.Boris, N.T. Janna, Analytica Chimica Acta 1997,347-351

20. D.Barcelo, S.Lacorte, J.L.Marty, Trends in Analytical chemistry, 1995,14,334-340

21. T.Marek, L. H.Michael, Trends in analytical chemistry, 1996,15,38-44

22. J.S.Hans, Journal of Chromatography A, 2000,892,347-377

23. Zh. Jiao, L.-F. Bian, J.-H. Liu, Chinese Journal of Functional Materials, 2001,32,67-68

24 V.I.Rigin, Anal.Chim.Acta, 1994,291, 121-125

25. A.J.Reviejo, A.Sampron, J.M.Pingarron, L.M.Polo, Electroanalysis, 1992,4,268-271

26. Zh.-Y. Zhang, Y.-H. Zhang, Zh. Jiao, J.-H.Liu, Chinese Journal of Sensors and Actuators, 2000,2, $106-110$

27. Zh.-Y. Zhang, J.-H. Liu, Y.-H. Zhang, Chinese Journal of Sensors and Actuators, 2000,1,13-17

28. W.M.Sears, K.Colbow, F.Consadori, Semicond.Sci.Technol. 1989,4,351-359

29. W.M.Sears, K.Colbow, F.Consadori, Sens.Actuators 1989,19,333-349

Sample Availability: Available from the authors.

(C) 2003 by MDPI (http://www.mdpi.net). Reproduction is permitted for noncommercial purposes. 